\title{
Cervical Spine Inhomogeneously Enhancing Lesion: Avoiding Confirmation Bias
}

\author{
Federica Penner ${ }^{1, *}$ Pietro Zeppa ${ }^{1, *}$ Fabio Cofano ${ }^{1}$ Andrea Bianconi ${ }^{1}$ Marco Ajello ${ }^{1}$ \\ Francesco Zenga ${ }^{1}$ \\ ${ }^{1}$ Section of Neurosurgery, Division of Neuroscience, University of \\ Turin, Italy \\ Address for correspondence Andrea Bianconi, MD, Dipartimento di \\ Neuroscienze, via Cherasco 15, 10126, Torino, Italy 3475805577 \\ J Neurosci Rural Pract 2022;13:134-136. \\ (e-mail: andrea.bianconi@edu.unito.it).
}

\begin{abstract}
Keywords

- cervical disc herniation

- cervical meningioma

- confirmation bias

- partial corpectomy

Confirmation bias is the tendency to seek information and evidence in order to confirm a preexisting hypothesis while giving less importance and overlook an alternative solution. This report describes the case of a 52-year-old man with a long history of neck pain and bilateral upper limbs paresthesias with a cervical intracanal inhomogeneously enhancing lesion. Despite all the preoperative radiological findings, a spinal meningioma an anterior approach was performed. The mass ended up being a large migrated hernia with the involvement of two levels. Before suggesting treatment, especially surgery, physicians and practitioners need to evaluate all of the possible alternatives in order to optimize patient outcome.
\end{abstract}

\section{Introduction}

Confirmation bias is the tendency to seek evidence by favoring an idea in order to confirm a preexisting hypothesis. This type of bias can be arduously hindering and misleading, resulting in a poor diagnosis and ultimately incorrect treatment. Furthermore, if an initial theory is inaccurate, it might drastically complicate the possibility of considering other hypothesis that could potentially lead to more beneficial results. This article describes a clinical case in which a more conventional interpretation of some radiological elements led to a misdiagnosis that might have been avoided in a patient with a cervical intracanal inhomogeneously enhancing lesion.

\footnotetext{
* The authors equally contributed to this manuscript.
}

published online January 11, 2022
DOI https://doi.org/ 10.1055/s-0041-1741489. ISSN 0976-3147.

\section{Case Report}

\section{Clinical Presentation}

A 52-year-old man with a long history of neck pain and bilateral upper limbs paresthesias was seen in the outpatient neurosurgical clinic.

Approximately 4 months prior to neurosurgical evaluation, the patient's neck pain substantially worsened and began to radiate to both arms. Oral use of nonsteroidal anti-inflammatory disease did, however, provide slight relief. A magnetic resonance imaging (MRI) showed an intracanal lesion adherent to the posterior wall of the C6-C7 vertebras. This finding inhomogeneously enhanced after gadolinium was administered. The radiological diagnosis provided was a spinal meningioma. Because of the (c) 2022. Association for Helping Neurosurgical Sick People. All rights reserved.

This is an open access article published by Thieme under the terms of the Creative Commons Attribution-NonDerivative-NonCommercial-License, permitting copying and reproduction so long as the original work is given appropriate credit. Contents may not be used for commercial purposes, or adapted, remixed, transformed or built upon. (https://creativecommons.org/ licenses/by-nc-nd/4.0/)

Thieme Medical and Scientific Publishers Pvt. Ltd., A-12, 2nd Floor, Sector 2, Noida-201301 UP, India 


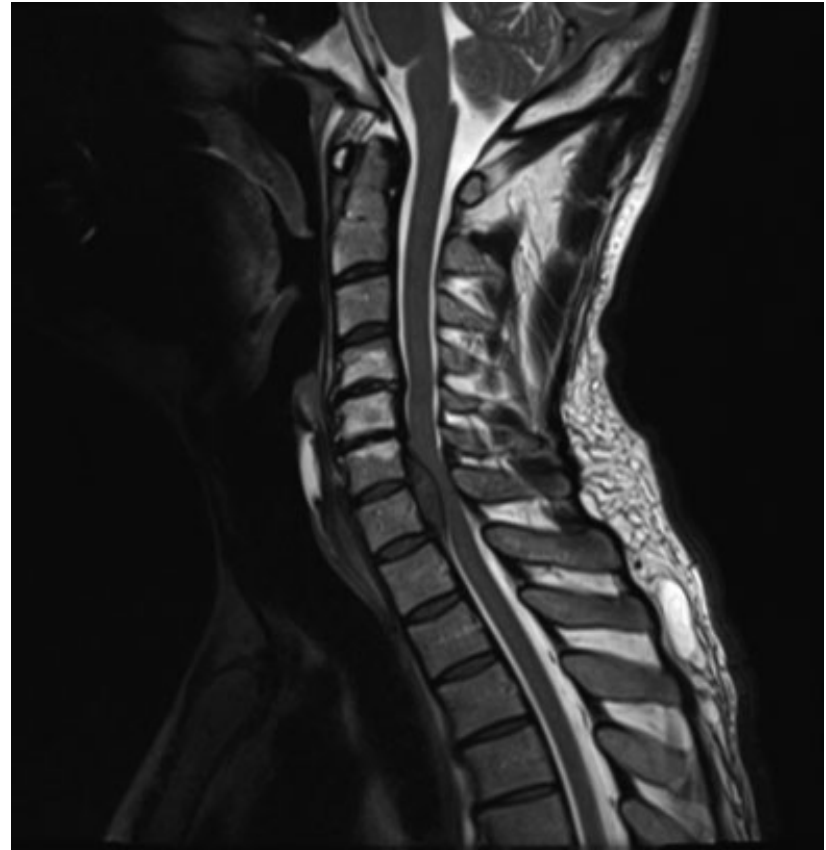

Fig. 1 T2-weighted sagittal view showing the lesion.

radiological and clinical diagnosis, no additional pharmacological treatment, that is, steroids, was suggested in order to mitigate the symptoms.

The risks and benefits of both the conservative and the surgical treatment were explained to the patient and thereafter it was unanimously decided to undergo microsurgical resection of the mass.

\section{Radiological Findings}

A second MRI study was obtained during admission. It showed proper alignment of the vertebral bodies: multiple protrusions of the $\mathrm{C} 4-\mathrm{C} 5, \mathrm{C} 5-\mathrm{C} 6, \mathrm{C} 6-\mathrm{C} 7$, and C7-D1 discs as well as a Modic 3 degeneration of the vertebral bodies at the above-mentioned levels. Also, it highlighted the presence of an apparently intradural mass, with a disc-like signal, posteriorly to the $\mathrm{C} 6$ and $\mathrm{C} 7$ bodies that compressed the spinal cord. There was no intramedullary signal alteration ( - Fig. 1).

After injecting medium contrast, there was a tenuous impregnation on the surface of the lesion that seemed to be in continuity with the dura mater (-Fig. 2).

The MRI findings were compatible with the presence of a meningioma.

\section{Surgery}

The day after admission, the patient underwent surgery. Because of the unusual MRI sign of the mass placed on the anterior segment of the cervical canal, occupying two spaces, an anterior approach was chosen. A linear left lateral neck incision at the level of $\mathrm{C} 6-\mathrm{C} 7$ disc was performed. A microdiscectomy was conducted. At this point, it was evident that what was thought to be a meningioma was in fact an extruded disc. The material was extradural fibrous tissue that was sent for pathological anatomy examination. In order to safely remove the hernia, it was decided to go on with a

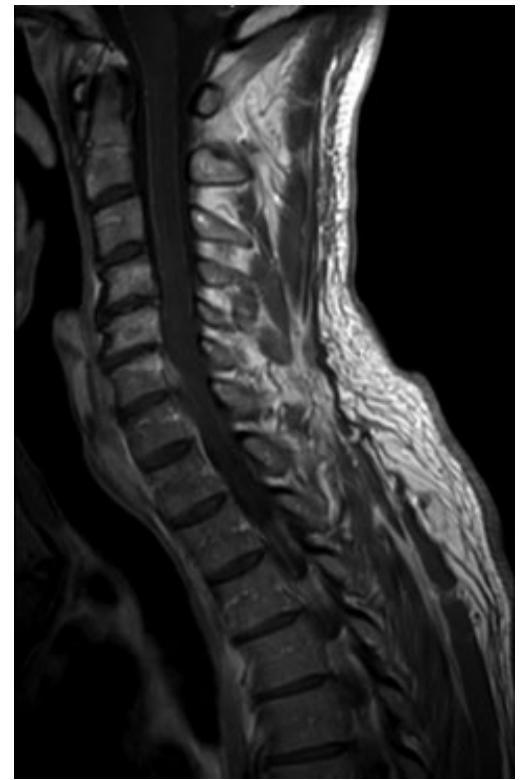

Fig. 2 T1-weighted contrast-enhanced view showing the inhomogeneous enhancement with apparent dural involvement.

partial corpectomy of cranial soma of the $\mathrm{C7}$ with a $\mathrm{C} 6-\mathrm{C} 7$ segment fusion. Also, an allograft was placed in the C6-C7 space and then secured with an anterior plate and some screws to enhance stability and fusion. By doing so, preservation of motion of an extra segment was possible. No surgical complication occurred. His admission was free from complication and he was discharged with a cervical Philadelphia collar on postsurgical day 2 .

A few days later, the pathological evaluation report was available. The specimen was fibrous-cartilaginous tissue with hyaline degeneration aspects; therefore, it was a migrated herniated discs probably from the levels above the C6.

\section{Discussion}

Meningiomas are usually a well-circumscribed mass lesion with large dura attached. They are hyperdense on computed tomography scans, isohypointense on T1-weighted, and isohyperintense on T2-weighted MRIs. Calcifications may be seen, but they are less common than in the intracranial meningiomas and occur in less than $5 \%$ of patients. ${ }^{1}$ Characteristically, meningiomas show strong homogeneous enhancement after medium contrast administration. ${ }^{2}$ Dura mater next to the attachment usually enhances. This sign, called dura tail, may be helpful in the diagnosis of spinal meningioma, although it is not specific. ${ }^{3}$ Meningiomas can cause compression and displacement of the spinal cord, but signal changes in the medulla secondary to compression are rarely seen.

The MRI findings of our patient were compatible with a meningioma, although the impregnation was not so homogeneous as one might expect.

Herniated discs are not so different from meningiomas on an MRI. They are usually hypointense on T1-weighted images 
and hyperintense on T2-weighted images. After gadolinium administration, they can show peripheral enhancement. The tenuous impregnation seen on the surface of our patient's mass lesion was therefore compatible with a herniated and migrated disc.

In this specific case, surgery facilitated in a definitive diagnosis of both illnesses and yielded relief from all of the symptoms. However, it is not possible to know if the symptoms could have been resolved solely by medical therapy. Furthermore, a herniated disc generally retreats naturally towards dehydration and shrinks within about 3 moths. ${ }^{4}$ For those who do not respond to medical therapy, surgery is advised. In this case, the patient waited more than 3 months before considering neurosurgical evaluation and his symptoms only worsened. However, it must be underlined that physician-supervised medical therapy was not initiated. It is likely that surgery would have been suggested also if the diagnosis of cervical hernia was taken into account, but it is most probable that medical therapy would have been added. The anterior approach allowed the surgeon to leave the dura intact once the origin of the mass was determined. A partial corpectomy with a bone graft permitted the complete removal of the mass with the necessity of one level of interbody fusion. This approach allowed to maintain a correct cervical alignment considering that a degenerative spinal disc pathology was faced. $^{5}$

\section{Conclusions}

First impressions are difficult to face while challenging to deal with to say the least. However, it is necessary to stop and reconsider all of the possible diagnosis before performing surgical procedures. Additionally, it is easy to overlook alternative solutions, thus complicating taking into account various diagnosis for specific symptoms. Moreover, we often place biases based on our experiences and by previous specialist indications. In this case, if the more common posterior approach for a cervical meningioma would have been used, the dura would have been opened for no reason exposing the patient to unnecessary risks. In literature, there are some cases in which the confirmation bias was actually harmful to patients, although in areas other than neurosurgery. ${ }^{6,7}$ Therefore, before suggesting treatment, especially if invasive as surgery usually is, physicians and practitioners need to evaluate all of the possible alternatives in order to optimize patient outcome. Following such protocol has potential to improve patient health while improving our own medical knowledge.

\section{Video 1}

T2- weighted MRI sequence. Online content including video sequences viewable at: https://www. thieme-connect.com/products/ejournals/html/ 10.1055/s-0041-1741489.

\section{Video 2}

T1- weighted contrast enhanced MRI sequence. Online content including video sequences viewable at: https:// www.thieme-connect.com/products/ejournals/html/ 10.1055/s-0041-1741489.

Conflict of Interest

None declared.

\section{References}

1 Pinter NK, Pfiffner TJ, Mechtler LL. Neuroimaging of spine tumors. In: Handbook of Clinical Neurology. 2016: Vol 136, Chapter 33, 689-706

2 Kunimatsu A, Kunimatsu N, Kamiya K, Katsura M, Mori H, Ohtomo K. Variants of meningiomas: a review of imaging findings and clinical features. Jpn J Radiol 2016;34(07):459-469

3 Tokumaru A, O'uchi T, Eguchi T, et al. Prominent meningeal enhancement adjacent to meningioma on Gd-DTPA-enhanced MR images: histopathologic correlation. Radiology 1990;175 (02):431-433

4 Mochida K, Komori H, Okawa A, Muneta T, Haro H, Shinomiya K. Regression of cervical disc herniation observed on magnetic resonance images. Spine 1998;23(09):990-995, discussion 996-997

5 Ajello M, Marengo N, Pilloni G, et al. Is it possible to evaluate the ideal cervical alignment for each patient needing surgery? An easy rule to determine the appropriate cervical lordosis in preoperative planning. World Neurosurg 2017;97:471-478

6 Jafferji D, Morris R, Levy N. Reducing the risk of confirmation bias in unrecognised oesophageal intubation. Br J Anaesth 2019;122 (04):e66-e68

7 Pang D, Bleetman A, Bleetman D, Wynne M. The foreign body that never was: the effects of confirmation bias. Br J Hosp Med (Lond) 2017;78(06):350-351 